\title{
Fractional Er:YAG laser assisting topical betamethasone solution in combination with NB-UVB for resistant non-segmental vitiligo
}

\author{
Ru Yan ${ }^{1,2} \cdot$ Jinping Yuan ${ }^{1} \cdot$ Hongqiang Chen ${ }^{3}$ Yuan-Hong $\mathrm{Li}^{1} \cdot \operatorname{Yan} \mathrm{Wu}^{1}{ }^{1}$ - \\ Xing-Hua Gao ${ }^{1} \cdot$ Hong-Duo Chen ${ }^{1}$
}

Received: 18 February 2016 / Accepted: 5 July 2017 / Published online: 14 July 2017

(C) The Author(s) 2017. This article is an open access publication

\begin{abstract}
Resistant non-segmental vitiligo is difficult to be treated. Ablative erbium-YAG (Er:YAG) laser has been used in the treatment of vitiligo, but the ablation of entire epidermis frustrated the compliance of patients. The purpose of this study is to investigate the effects of fractional Er:YAG laser followed by topical betamethasone and narrow band ultraviolet B (NB-UVB) therapy in the treatment of resistant nonsegmental vitiligo. The vitiligo lesions of each enrolled patient were divided into four treatment parts, which were all irradiated with NB-UVB. Three parts were, respectively, treated with low, medium, or high energy of Er:YAG laser, followed by topical betamethasone solution application. A control part was spared with laser treatment and topical betamethasone. The treatment period lasted 6 months. The efficacy was assessed by two blinded dermatologists. Treatment protocol with high energy of $1800 \mathrm{~mJ} / \mathrm{P}$ of fractional Er:YAG laser followed by topical betamethasone solution and in combination with NB-UVB made $60 \%$ patients achieve marked to excellent improvement in white patches. The protocol with medium energy of $1200 \mathrm{~mJ} / \mathrm{P}$ of laser assisted approximate $36 \%$ patients achieve such improvement. The two protocols, respectively, showed better efficacies than NB-UVB only
\end{abstract}

Yan $\mathrm{Wu}$

jlwuyan@126.com

Xing-Hua Gao

gaobarry@hotmail.com

1 Department of Dermatology, No.1 Hospital of China Medical University, 155 North Nanjing Street, Shenyang 110001, China

2 Department of Dermatology, Affiliated Hospital of Jining Medical University, Jining, China

3 Department of General Surgery, Shandong Qianfoshan Hospital, Jinan, China protocol. However, fractional Er:YAG laser at low energy of $600 \mathrm{~mJ} / \mathrm{P}$ did not provide such contributions to the treatment of vitiligo. The fractional Er:YAG laser in combination with topical betamethasone solution and NB-UVB was suitable for resistant non-segmental vitiligo. The energy of laser was preferred to be set at relatively high level.

Keywords Vitiligo $\cdot$ Er:YAG laser $\cdot$ Fractional

Vitiligo is a common acquired pigmentary disorder of the skin and mucous membranes. It has significant effects on quality of life and remains a persistent burden for the patients $[1,2]$. Traditional treatments for vitiligo included topical or systematic medication, photo(chemo)therapy, surgery, camouflage, et al. [3]. However, seldom of them gives satisfactory results in refractory vitiligo. Thus, combination treatments incorporating conventional modalities and new therapeutic approaches are required.

There have been several reports introducing ablative laserassisted trans-epidermal delivery of topical medicine in the treatment of vitiligo [4-6]. The ablative lasers including carbon dioxide $\left(\mathrm{CO}_{2}\right)$ laser and erbium-YAG (Er:YAG) laser were considered superior to mechanical dermabrasion, owing to the controlled ablation $[7,8]$. The effects of $\mathrm{CO}_{2}$ laser or Er:YAG laser ablation followed by topical application of 5fluorouracil (5-FU) or hydrocortisone 17-butyrate cream combined with narrow band ultraviolet B (NB-UVB) phototherapy or not were, respectively, reported for treating nonsegmental vitiligo [9-11]. However, ablation of the entire epidermis frustrated the skin healing process.

In 2004, fractional photothermolysis was introduced by Manstein and colleagues as a new concept for laser skin rejuvenation. Fractional laser do not ablate the entire epidermis and thereafter leave intact skin between coagulated necrotic 
columns [8]. This characteristic facilitates the skin healing process. Recently, fractional $\mathrm{CO}_{2}$ laser was reported to be used in the treatment of refractory vitiligo followed by NB-UVB or sun exposure $[12,13]$. In 2015, we reported a triple combination treatment with fractional $\mathrm{CO}_{2}$ laser assisting delivery of topical betamethasone solution plus NB-UVB phototherapy for refractory vitiligo and achieved some preliminary and encouraging results [14].

Fractional laser with the Er:YAG laser was introduced as a gentler alternative to the $\mathrm{CO}_{2}$ laser. Under proper parameter setting, the Er:YAG laser could create less residual thermal damage and faster healing [8, 12]. In the present study, we investigated the effects of fractional Er:YAG laser followed by topical application of betamethasone solution and NB-UVB on resistant non-segmental vitiligo. We provided an alternative choice for the treatment of vitiligo.

\section{Materials and methods}

\section{Patients}

All procedures performed in the study were in accordance with the ethical standards of the Institutional Medical Ethics and Human Research Committee and with the 1964 Helsinki declaration and its later amendments or comparable ethical standards. Patients with more than 1 year of stable, nonsegmental and resistant vitiligo were recruited. The resistant vitiligo was defined as poor response to conventional treatments, including topical or systematic medication, phototherapy, or surgery. The age ranged from 18 to 60 . Patients who received any treatments within 3 months prior to enrollment or had other dermatological or systemic disorders were excluded. Pregnancy or lactation females were excluded.

\section{Treatment protocols}

Vitiligo patches of each patient were equally divided to four parts to receive different treatment protocols. Fractional ablative photothermolysis treatment was carried out using a 2940-nm Er:YAG laser (Pixel, Alma Lasers Ltd., Caesarea, Israel) that incorporates a microlens aligned in a matrix of $9 \times 9(81)$ dots (pixels), which emits $17 \mathrm{~mJ} / \mathrm{P}$ per pixel. The single-pass ablation microzone of each pixel measures approximately $150 \mathrm{~mm}$ in diameter and $120-140 \mathrm{~mm}$ in depth. The pulse widths of the device were preset at short, medium, and long. Three parts of white patches were monthly treated with the fractional $2940 \mathrm{~nm}$ Er:YAG laser at energy of 600, 1200 , and $1800 \mathrm{~mJ} / \mathrm{P}$, respectively. The 600,1200 , and $1800 \mathrm{~mJ} / \mathrm{P}$ in the pulse referred to the energy over the "entire larger spot" and not the "micro spot" $(17 \mathrm{~mJ} / \mathrm{P})$. Two stacked laser passes were performed and the pulse width was set at medium. Immediately after the laser treatment, compound betamethasone solution (Schering Pharmaceutical Company Limited, Shanghai, China) was topically applied under occlusion with a plastic film for 30 min. One part, as a positive control, was spared with laser treatment and topical betamethasone. A NB-UVB instrument (SS03, Shanghai Sigma High-tech Co., Ltd., Shanghai, China) was used for phototherapy to all the four parts according to previous description [14]. Briefly, the phototherapies were performed twice to thrice a week, beginning at a dose of $100-150 \mathrm{~mJ} / \mathrm{cm}^{2}$ and elevating by approximately $10-15 \%$ each time (up to a maximum of $1500 \mathrm{~mJ} / \mathrm{cm}^{2}$ ). A summary of the treatment was given in Table 1. The total period of treatment was 6 months.

\section{Measurement}

Standard photos were taken with a digital camera (D40S, Nikon Corporation, Tokyo, Japan) at baseline (M0) and 1 month after each laser treatment. Three months posttreatment (M3) and 6 months post-treatment (M6), the response to the treatment was assessed by two blinded dermatologists. Depending on the extent of re-pigmentation, the response to the treatment was graded as: no or minimal $(<25 \%)$, moderate $(25-49 \%)$, marked $(50-74 \%)$, excellent $(\geq 75 \%)[12,13]$. Any side effect was recorded during the procedure.

\section{Statistic analysis}

Data were analyzed using SPSS for software (version 20.0, SPSS Inc., Chicago, IL, USA). Comparisons were performed by Kruskal-Wallis Test or Mann-Whitney analysis. A $P$ value $<0.05$ was considered as statistically significant.

\section{Results}

\section{Patients}

A total of 22 patients completed the study, including 10 men $(45 \%)$ and 12 women (55\%). The mean age was 48 years (range 21-60). Thirty-five paired vitiligo lesions were involved in different body sites: 6 on the neck, 10 on the trunk, 11 on the anterior region of leg, and 8 on the hands or feet. Figures 1, 2, and 3 showed the representative photographs of the patients pre- and post-treatments. Table 2 presents the patients' responses to different treatment protocols. 
Table 1 Treatment description of each protocol

\begin{tabular}{|c|c|c|c|c|}
\hline & Protocol 1 & Protocol 2 & Protocol 3 & Protocol 4 \\
\hline $\begin{array}{l}\text { Fractional } \\
\quad \text { Er:YAG laser }\end{array}$ & - & low energy $(600 \mathrm{~mJ})$ & medium energy $(1200 \mathrm{~mJ})$ & $\begin{array}{l}\text { high energy } \\
\quad(1800 \mathrm{~mJ})\end{array}$ \\
\hline $\begin{array}{l}\text { Betamethasone } \\
\text { solution }\end{array}$ & - & + & + & + \\
\hline NB-UVB & + & + & + & + \\
\hline
\end{tabular}

\section{The overall comparison of four protocols}

The four protocols produced significantly different responses to the treatments, at whatever M3 $(\chi 2=19.501, P<0.001)$ or M6 $(\chi 2=19.716, P<0.001)$. No significant difference was seen between protocol 2 and protocol 1at either M3 $(P=0.300)$ or M6 $(P=0.196)$. The overall response to therapy of protocol 3 was significantly better than that of protocol $1(\mathrm{M} 3, \chi 2=6.689$, $P=0.010 ; \mathrm{M} 6, \chi 2=6.142, P=0.013)$. The protocol 4 was also better than protocol 1 (M3, $\chi 2=14.710, P<0.001$; M6, $\chi 2=16.106, P<0.001)$. No difference was seen between protocol 3 and protocol 4 at M3 $(P=0.113)$ or M6 $(P=0.078)$.

\section{The comparison of M6 with M3}

The response of M6 was significantly better than that of M3 treated by protocol $2(Z=2.084, P=0.037)$, protocol 3
$(Z=1.976, P=0.048)$, or protocol $4(Z=3.014, P=0.003)$. The results of protocol 1at M6 and at M3showed no statistical difference $(P=0.079)$.

\section{The various responses to four protocols at different sites}

Different sites, including the neck, trunk, and the anterior region of leg and hands/feet, showed various responses to the four protocols. Table 3 showed the detailed statistic results.

On the trunk and the anterior region of leg, four protocols produced significant different responses at whatever M3 or M6 $(P<0.05)$. Protocol 3 and protocol 4 showed better efficacy than protocol 1 , respectively $(P<0.05)$. However, no significant difference was seen between protocol 2 and protocol $1(P>0.05)$.
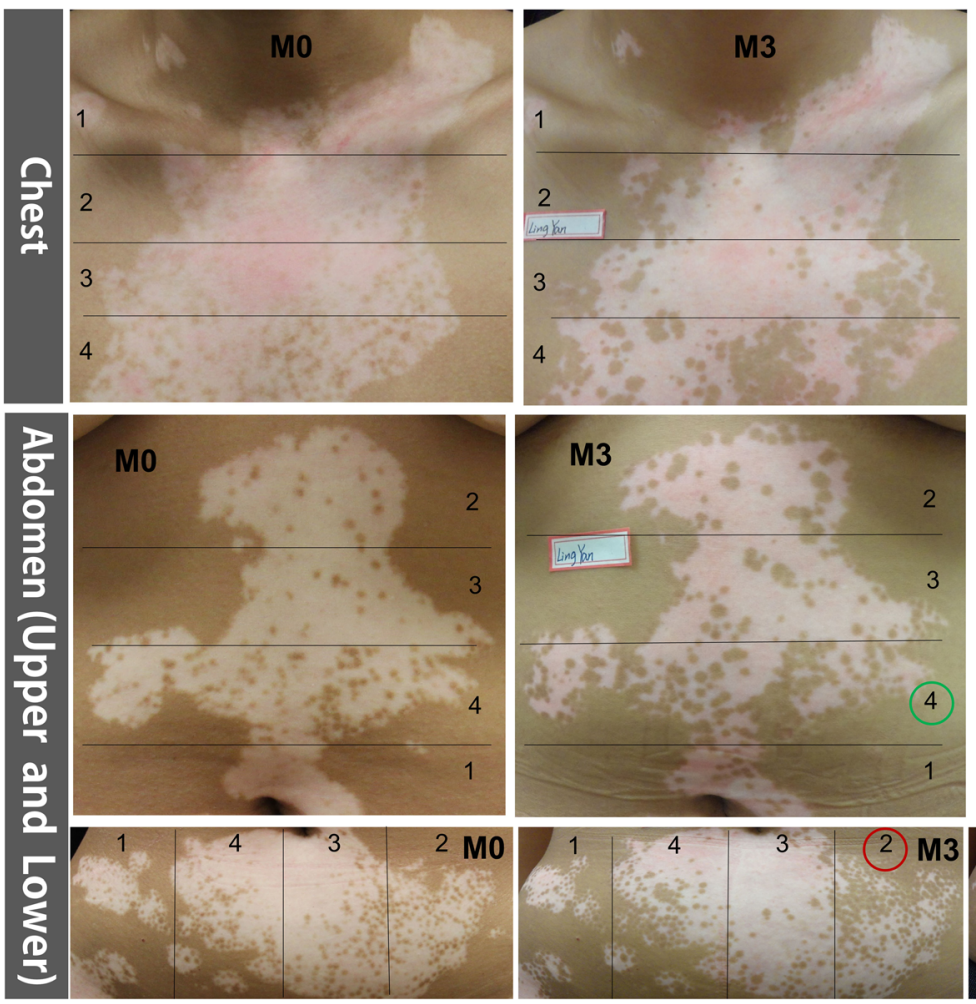
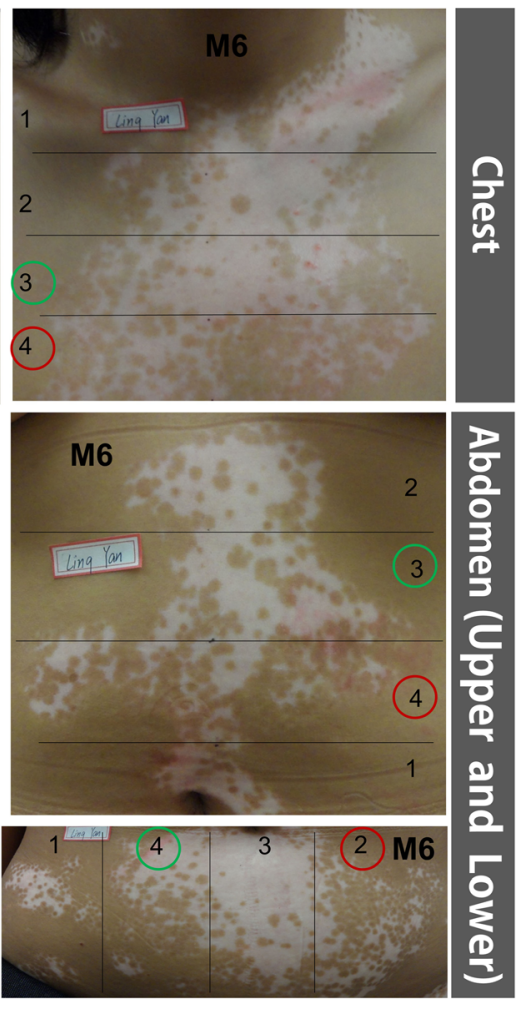

Fig. 1 The pre- and post-treatment photographs on the chest and the abdomen of a 36-year-old female vitiligo patient. Note: $M 0$, pretreatment; $M 3,3$ months post-treatment; $M 6,6$ months post-treatment; the Arabic numerals 1,2,3, and 4 were referred to as protocols 1,2,3, and
4, respectively. Red circle of the Arabic numeral meant excellent $(\geq 75 \%)$ response to treatment; green circle of the Arabic numeral meant marked $(50-74 \%)$ response to treatment 
Fig. 2 The pre- and posttreatment photographs on the hands and the anterior regions of two legs of a 42-year-old female vitiligo patient. Note: $M 0$, pretreatment; $M 3,3$ months posttreatment; M6, 6 months posttreatment; the Arabic numerals 1 , 2,3 , and 4 were referred to as protocols $1,2,3$, and 4 , respectively. Red circle of the Arabic numeral meant excellent $(\geq 75 \%)$ response to treatment; green circle of the Arabic numeral meant marked (50-74\%) response to treatment

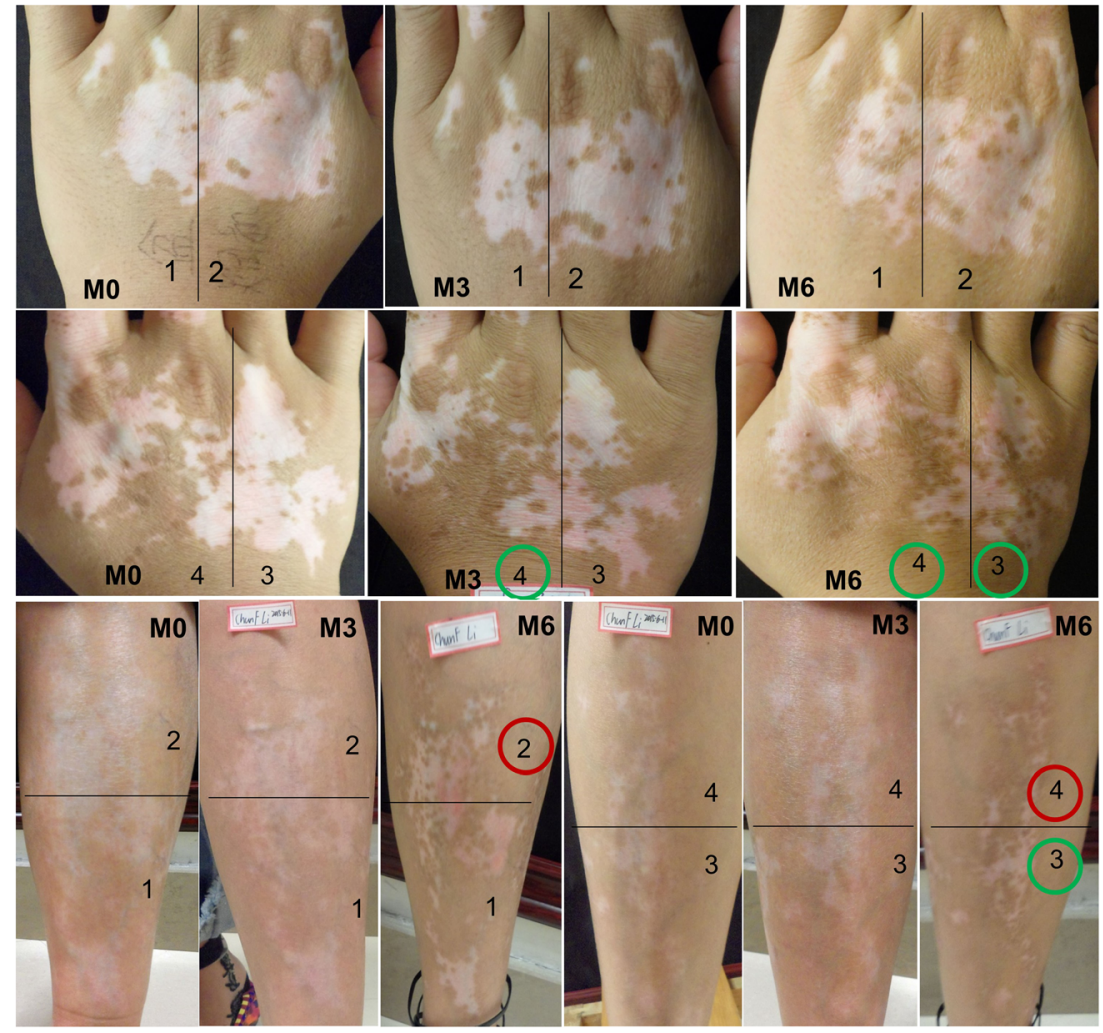

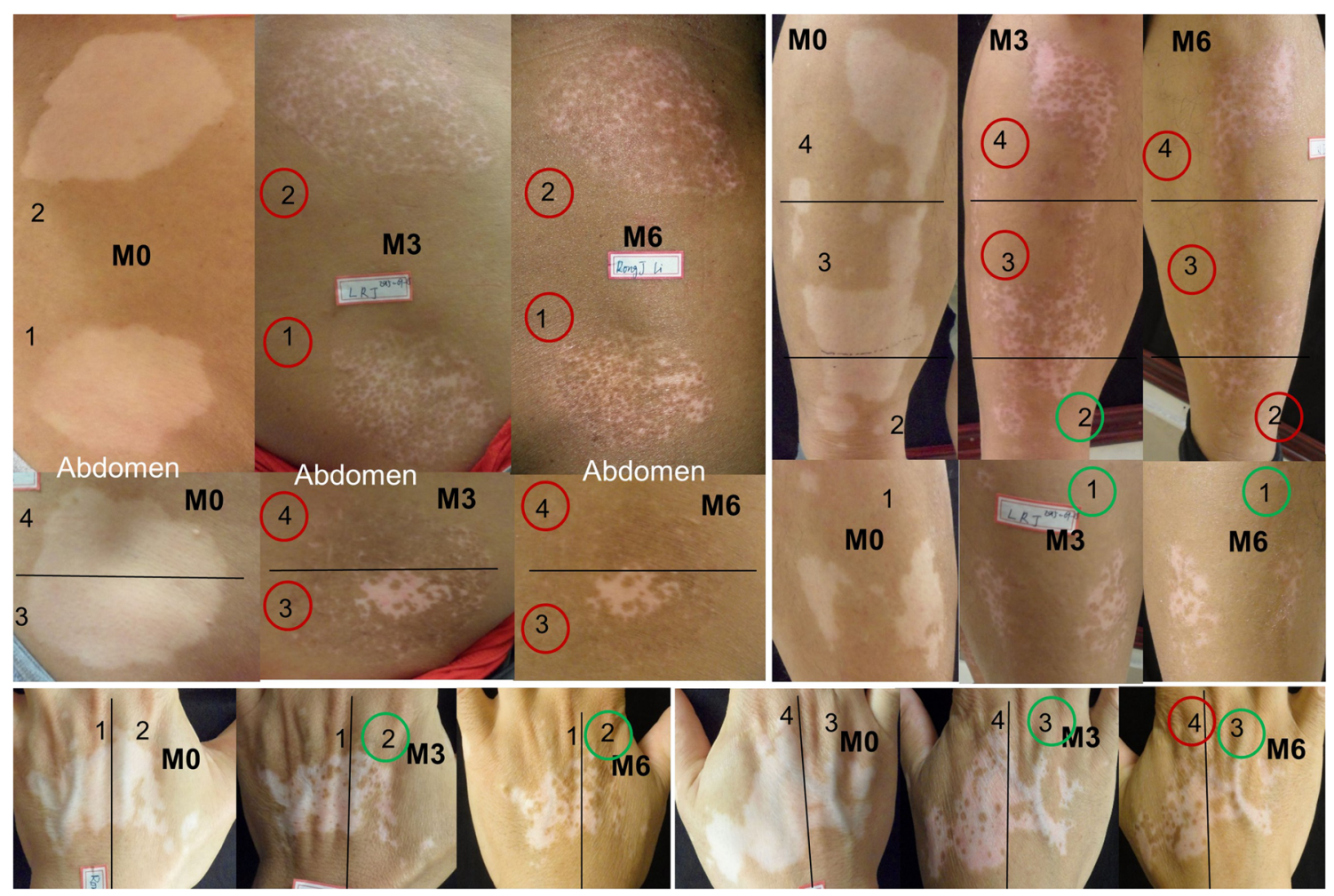

Fig. 3 The pre- and post-treatment photographs on the abdomen, the anterior regions of two legs and the hands of a 60-year-old male vitiligo patient. Note: $M 0$, pre-treatment; $M 3,3$ months post-treatment; $M 6$, 6 months post-treatment; the Arabic numerals 1,2,3, and 4 were referred to as protocols $1,2,3$, and 4, respectively. Red circle of the Arabic numeral meant excellent $(\geq 75 \%)$ response to treatment; green circle of the Arabic numeral meant marked $(50-74 \%)$ response to treatment 
Table 2 The patients' responses to different treatment protocols ( $n$ (\%))

\begin{tabular}{lllll}
\hline & Protocol 1 & Protocol 2 & Protocol 3 & Protocol 4 \\
\hline $\begin{array}{l}\text { Three months post-treatment } \\
\text { No or minimal }(<25 \%)\end{array}$ & $21(95.45 \%)$ & $19(86.36 \%)$ & $14(63.64 \%)$ & $9(40.91 \%)$ \\
Moderate $(25-49 \%)$ & $1(4.55 \%)$ & $3(13.64 \%)$ & $8(36.36 \%)$ & $12(54.54 \%)$ \\
Marked $(50-74 \%)$ & $0(0 \%)$ & $0(0 \%)$ & $0(0 \%)$ & $1(4.55 \%)$ \\
Excellent $(\geq 75 \%)$ & $0(0 \%)$ & $0(0 \%)$ & $0(0 \%)$ & $0(0 \%)$ \\
Six months post-treatment & & & & \\
No or minimal $(<25 \%)$ & $17(77.27 \%)$ & $13(59.09 \%)$ & $10(45.45 \%)$ & $5(22.73 \%)$ \\
Moderate $(25-49 \%)$ & $4(18.18 \%)$ & $7(31.81 \%)$ & $4(18.18 \%)$ & $4(18.18 \%)$ \\
Marked $(50-74 \%)$ & $1(4.55 \%)$ & $1(4.55 \%)$ & $6(27.27 \%)$ & $8(36.36 \%)$ \\
Excellent $(\geq 75 \%)$ & $0(0 \%)$ & $1(4.55 \%)$ & $2(9.09 \%)$ & $5(22.73 \%)$ \\
\hline
\end{tabular}

On both the neck or hands/feet portion, however, no difference was seen among the four protocols at whatever M3 or M6 $(P>0.05)$.

\section{Safety evaluation}

No patients developed noticeable side effects, including local infection, scarring, Koebner phenomenon, and aggravation of vitiligo. All patients experienced slight pain and burning sensation during the laser treatment, as well as erythema and slight edema after laser treatment. The symptoms were tolerable and relieved within a day. About 50\% patients developed micro-crust on the area with high energy $(1800 \mathrm{~mJ} / \mathrm{P})$ of laser treatment, and disappeared within 3-5 days.

\section{Discussion}

In recent years, various combination treatments have been attempted in an effort to enhance efficacy in patients with resistant vitiligo [5]. New treatment approaches such as using ablative lasers followed by topical application of 5-FU or steroids combined with NBUVB phototherapy were studied and increased the repigmentation rate of vitiligo [9, 10]. Despite their promising effects of the ablative lasers, ablation of the entire epidermis raises difficulties in the regulation of resurfacing depth and wound care, and involves a high risk of potential scars. In 2004, fractional laser therapy was developed to reduce the side effects of total ablative lasers. Recently, the fractional $\mathrm{CO}_{2}$ laser followed by topical steroids combined with NB-UVB or sun exposure has been proved to be effective in the treatment of refractory vitiligo $[12,13]$. In 2015 , we also reported a triple combination treatment with fractional $\mathrm{CO}_{2}$ laser assisting delivery of topical betamethasone solution plus NB-UVB phototherapy for refractory vitiligo and achieved some encouraging results [14]. Till now, another type of fractional ablative laser, i.e., fractional Er:YAG laser has not been investigated in the treatment of vitiligo.

In this study, the protocols treating with high energy $(1800 \mathrm{~mJ} / \mathrm{P})$ or medium energy $(1200 \mathrm{~mJ} / \mathrm{P})$ of fractional
Table 3 The comparison of the four protocols at different sites

\begin{tabular}{|c|c|c|c|c|c|c|c|c|}
\hline & \multicolumn{2}{|l|}{ Neck } & \multicolumn{2}{|l|}{ Trunk } & \multicolumn{2}{|c|}{$\begin{array}{l}\text { Anterior region of } \\
\text { leg }\end{array}$} & \multicolumn{2}{|c|}{ Hands/feet } \\
\hline & $\chi^{2}$ & $P$ & $\chi^{2}$ & $P$ & $\chi^{2}$ & $P$ & $\chi^{2}$ & $P$ \\
\hline \multicolumn{9}{|c|}{ Three months post-treatment } \\
\hline Overall comparison & 2.332 & 0.506 & 15.239 & $0.002 *$ & 14.587 & $0.002 *$ & 0.969 & 0.809 \\
\hline Protocol 2 vs. protocol 1 & 0.314 & 0.575 & 3.736 & 0.053 & 1.167 & 0.280 & 0.292 & 0.589 \\
\hline Protocol 3 vs. protocol 1 & 0.815 & 0.367 & 7.246 & $0.007 *$ & 5.091 & $0.024 *$ & 0.292 & 0.589 \\
\hline Protocol 4 vs. protocol 1 & 1.956 & 0.162 & 12.095 & $0.001 *$ & 11.094 & $0.001 *$ & 0.937 & 0.333 \\
\hline \multicolumn{9}{|c|}{ Six months post-treatment } \\
\hline Overall comparison & 6.112 & 0.106 & 10.543 & $0.014 *$ & 11.580 & $0.009 *$ & 2.705 & 0.439 \\
\hline Protocol 2 vs. protocol 1 & 0.399 & 0.527 & 2.609 & 0.106 & 0.524 & 0.469 & 0.380 & 0.538 \\
\hline Protocol 3 vs. protocol 1 & 2.842 & 0.092 & 5.417 & $0.020^{*}$ & 6.056 & $0.014 *$ & 1.221 & 0.269 \\
\hline protocol 4 vs. protocol 1 & 4.694 & $0.030 *$ & 8.205 & $0.004 *$ & 7.429 & $0.006^{*}$ & 2.368 & 0.124 \\
\hline
\end{tabular}


Er:YAG laser produced significantly better responses comparing to the protocols treating with low energy $(600 \mathrm{~mJ} /$ P) of laser or NB-UVB only. At M3, at least $25 \%$ repigmentation was, respectively, seen on approximate 36 and $60 \%$ patients under the former protocols. At M6, the two protocols enabled about 55 and $77 \%$ patients achieve such re-pigmentation, and about 36 and $60 \%$ patients achieve at least $50 \%$ re-pigmentation. The results indicated that fractional Er:YAG laser did provide additional contribution to the treatment of vitiligo, but the energy should be set at the level of at least $1200 \mathrm{~mJ}$. Although no significant difference was seen between medium energy protocol and high energy protocol, the $P$ value of 0.078 at M6 almost approached the statistic difference. It implied that fractional Er:YAG laser at the high energy of $1800 \mathrm{~mJ}$ may produce better efficacy than the medium one. Increasing the patients' sample size may arise such results. The results of M6 were better than those of M3, suggesting a sufficient treatment period should be no less than 6 months.

Re-pigmentation in vitiligo is known to be initiated by activation, proliferation, and migration of melanoblasts from the outer root sheath of hair follicles or melanocytes from the border area of vitiligo lesions into the depigmented epidermis [15]. The mechanisms of repigmentation induced by our triple combination treatment protocol may be explained as follows: (1) the fractional Er:YAG laser treatment, which is performed over the vitiligo lesion, allows for resettlement of migrating melanocytes [4]; (2) the topical corticosteroid used here contains betamethasone disodiumphosphate (fast-acting) and betamethasone dipropionate (slow-acting) [14]. Aiding by the fractional Er:YAG laser, the compound formulation is expected to induce both rapid and lasting re-pigmentation responses [14]. (3) Matrix metalloproteinase-2 (MMP-2) is known to enhance migration of melanocytes from adjacent normal skin. Previous study showed that NB-UVB irradiation could significantly increase the activity of MMP-2 in melanocytes [16]. Dermabrasion using fractional Er:YAG laser followed by NB-UVB irradiation would enhance the stimulation of MMP-2 activity in vitiligo lesion.

In 2012, Bayoumi et al. used Er:YAG laser dermabrasion followed by NB-UVB and hydrocortisone 17-butyrate cream therapy to treat vitiligo for 3 months, and at least $50 \%$ re-pigmentation improvement was seen in $46 \%$ of lesions [10]. In our stud, using high energy of fractional Er:YAG laser, although such degree of re-pigmentation was only seen in $4.55 \%$ patients at M3, it was seen in almost $60 \%$ patients at M6. Despite the quick response and high rate of re-pigmentation of total ablative Er:YAG laser, the pain during the procedure and the slow healing process strongly impaired the tolerance and limited its use in current practice. In our study, no patients developed noticeable side effects, and all the patients were willing to continue the laser treatment with no need of anesthetic. Theoretically, fractional Er:YAG laser has the same mechanisms of re-pigmentation as ablative lasers, but can minimize the side effects.

In 2015, we reported a triple combination treatment with fractional $\mathrm{CO}_{2}$ laser assisting delivery of topical betamethasone solution plus NB-UVB phototherapy for refractory vitiligo [14]. In that study, over $50 \%$ re-pigmentation was seen on $40 \%$ patients at M3 and 44\% at M6. The results of the two time points were similar, indicating that three-month treatment was sufficient to induce the re-pigmentation response and continuous treatment to 6 months just maintained the efficacy. In our study using high energy laser, the patients who achieved at least $50 \%$ repigmentation significantly increased from $4.55 \%$ at $\mathrm{M} 3$ to about $60 \%$ at M6. Comparing to fractional $\mathrm{CO}_{2}$ laser, fractional Er:YAG laser was a gentler and "cooler" one. So the laser induced-response was relatively slower than the fractional $\mathrm{CO}_{2}$ laser, but continuous treatment to 6 months induced a comparative response. Although there were limitations in the present study, including the lack of a betamethasone-only control and a laser-only control, we did find that fractional Er:YAG laser may contribute to the treatment of vitiligo. Combination treatment with fractional Er:YAG laser plus topical betamethasone solution and NB-UVB therapy is suitable for resistant non-segmental vitiligo. The energy of laser is preferred to be set at relatively high level. More studies with large sample size are required to verify the results.

\section{Compliance with ethical standards}

Funding The study was supported by Liaoning Province Education Bureau Project (LZ2015077).

Conflict of interest The authors declare that they have no conflict of interest.

Informed consent Informed consent was obtained from all individual participants included in the study.

Ethical approval All procedures performed in the study were in accordance with the ethical standards of the Institutional Medical Ethics and Human Research Committee and with the 1964 Helsinki declaration and its later amendments or comparable ethical standards.

Ethical responsibilities of authors The manuscript has not been submitted to more than one journal for simultaneous consideration. The manuscript has not been published previously (partly or in full). A single study is not split up into several parts to increase the quantity of submissions and submitted to various journals or to one journal over time.

Open Access This article is distributed under the terms of the Creative Commons Attribution 4.0 International License (http:// creativecommons.org/licenses/by/4.0/), which permits unrestricted use, distribution, and reproduction in any medium, provided you give appropriate credit to the original author(s) and the source, provide a link to the Creative Commons license, and indicate if changes were made. 


\section{References}

1. Bae JM, Yoo HJ, Kim H et al (2015) Combination therapy with 308-nm excimer laser, topical tacrolimus, and short-term systemic corticosteroids for segmental vitiligo: a retrospective study of 159 patients. J Am Acad Dermatol 73:76-82. doi:10.1016/j.jaad.2015. 04.008

2. Speeckaert R, Speeckaert MM, van Geel N (2015) Why treatments do(n't) work in vitiligo: an auto inflammatory perspective. Autoimmun Rev 14:332-340. doi:10.1016/j.autrev.2014.12.003

3. Anbar TS, Hegazy RA, Picardo M et al (2014) Beyond vitiligo guidelines: combined stratified / personalized approaches for the vitiligo patient. Exp Dermatol 23:219-223. doi:10.1111/exd.12344

4. Anbar T, Westerhof W, Abdel-Rahman A et al (2006) Treatment of periungual vitiligo with erbium-YAG-laser plus 5-flurouracil: a left to right comparative study. J Cosmet Dermatol 5:135-139. doi:10. 1111/j.1473-2165.2006.00240.x

5. Garg T, Chander R, Jain A (2011) Combination of microdermabrasion and 5-fluorouracil to induce repigmentation in vitiligo: an observational study. Dermatol Surg 37:1763-1766. doi:10.1111/j.1524-4725.2011.02127.x

6. Sethi S, Mahajan BB, Gupta RR et al (2007) Comparative evaluation of the therapeutic efficacy of dermabrasion, dermabrasion combined with topical 5\% 5-fluorouracil cream, and dermabrasion combined with topical placentrex gel in localized stable vitiligo. Int $\mathrm{J}$ Dermatol 46:875-879. doi:10.1111/j.1365-4632.2007.03226.x

7. Sachdev M, Krupashankar DS Suction blister grafting for stable vitiligo using pulsed erbium:YAG laser ablation for recipient site. Int J Dermatol 39:471-473. doi:10.1046/j.1365-4362.2000.00989. $\mathrm{x}$

8. Manstein D, Herron GS, Sink RK et al (2004) Fractional photothermolysis: a new concept for cutaneous remodeling using microscopic patterns of thermal injury. Lasers Surg Med 34:426438. doi:10.1002/1sm. 20048

9. Anbar TS, Westerhof W, Abdel-Rahman AT et al (2008) Effect of one session of ER:YAG laser ablation plus topical 5Fluorouracil on the outcome of short-term NB-UVB phototherapy in the treatment of non-segmental vitiligo: a left-right comparative study. Photodermatol Photoimmunol Photomed 24:322-329. doi:10. 1111/j.1600-0781.2008.00385.x

10. Bayoumi W, Fontas E, Sillard L et al (2012) Effect of a preceding laser dermabrasion on the outcome of combined therapy with narrowband ultraviolet $\mathrm{B}$ and potent topical steroids for treating nonsegmental vitiligo in resistant localizations. Br J Dermatol 166:208-211. doi:10.1111/j.1365-2133.2011.10564.x

11. Mohamed HA, Mohammed GF, Gomaa AH et al (2015) Carbon dioxide laser plus topical 5-fluorouracil: a new combination therapeutic modality for acral vitiligo. J Cosmet Laser Ther 17:216-223. doi:10.3109/14764172.2014.1003241

12. Helou J, Maatouk I, Obeid G et al (2014) Fractional laser for vitiligo treated by $10,600 \mathrm{~nm}$ ablative fractional carbon dioxide laser followed by sun exposure. Lasers Surg Med 46:443-448. doi:10. $1002 / 1 \mathrm{sm} .22260$

13. Shin J, Lee JS, Hann SK et al (2012) Combination treatment by 10 $600 \mathrm{~nm}$ ablative fractional carbon dioxide laser and narrowband ultraviolet $\mathrm{B}$ in refractory nonsegmental vitiligo: a prospective, randomized half-body comparative study. Br J Dermatol 166:658-661. doi:10.1111/j.1365-2133.2011.10723.x

14. Li L, Wu Y, Li L et al (2015) Triple combination treatment with fractional $\mathrm{CO}$ laser plus topical betamethasone solution and narrowband ultraviolet $\mathrm{B}$ for refractory vitiligo: a prospective, randomized half-body, comparative study. Dermatol Ther 28:131-134. doi:10. 1111/dth.12202

15. Kumar R, Parsad D, Kanwar AJ et al (2011) Altered levels of Ets-1 transcription factor and matrix metalloproteinases in melanocytes from patients with vitiligo. Br J Dermatol 165:285-291. doi:10. 1111/j.1365-2133.2011.10324.x

16. Wu CS, Yu CL, Wu CS et al (2004) Narrow-band ultraviolet-B stimulates proliferation and migration of cultured melanocytes. Exp Dermatol 13:755-763. doi:10.1111/j.0906-6705.2004.00221.x 